\title{
Magnetic Resonance Imaging in Intracranial Tuberculosis - An Observational Study from Mangalore, India
}

\author{
Anvesh Apkari ${ }^{1}$, Rohini Avantsa르, Venkata Tejaswi ${ }^{3}$ \\ 1, 2, 3 Department of Radiodiagnosis, K.S. Hegde Medical Academy, \\ Nitte (Deemed to Be University), Mangalore, India.
}

\section{ABSTRACT}

\section{BACKGROUND}

Intracranial tuberculosis is one of the more severe forms of TB and an important cause of morbidity and mortality in affected individuals. It comprises $10 \%$ of total tuberculosis cases and $20 \%$ among immunocompromised patients. Contrastenhanced magnetic resonance imaging (MRI) is the imaging modality of choice, as it helps in its early diagnosis and to assess the complications of intracranial tuberculosis thereby reducing the morbidity and the mortality of the disease. The main objectives of this article were to revisit the spectrum of involvement and assess the tubercular lesions on MRI, determine the various presentations of intracranial tuberculosis and its associated complications.

\section{METHODS}

This is a single-center, hospital based, observational and retrospective study. The study included 25 patients who had proven intracranial tuberculosis and underwent MR examination of the brain from March 2015 to April 2020. All patients had been referred to the Department of Radiodiagnosis with clinical suspicion of intracranial pathology. The various manifestations and their complications were evaluated.

\section{RESULTS}

This present study comprised 15 males (60\%) and 10 females ( $40 \%$ ), having a male to female ratio of 1.5:1. The most common age group was $21-40$ years and $51-60$ years with a range of $16-68$ years. The 25 cases diagnosed with intracranial TB had a varied presentation. The most common presentation was isolated tuberculoma 15 $(60 \%)$ followed by mixed presentation 7 (28\%). Complications were seen in 5 (20 $\%)$, which included hydrocephalus, ventriculitis and infarcts.

\section{CONCLUSIONS}

Contrast enhanced MRI is the established modality of choice to diagnose and monitor intracranial tuberculosis. Good knowledge of its various imaging characteristics and spectrum of involvement helps in the diagnosis and management.

\section{KEY WORDS}

Tuberculosis, MRI, Tuberculoma
Corresponding Author:

Dr. Anvesh Apkari,

Villa Number 4 , Ashoka-Ala-Maison,

Dhulapally Road, Kompally,

Hyderabad, Telangana.

E-mail: apkarianvesh@gmail.com

DOI: $10.14260 /$ jemds/2021/495

How to Cite This Article:

Apkari A, Avantsa R, Tejaswi V. Magnetic resonance imaging in intracranial tuberculosis - an observational study from Mangalore, India. J Evolution Med Dent Sci 2021;10(31):2416-2421, DOI: 10.14260/jemds/2021/495

Submission 24-03-2021,

Peer Review 27-05-2021,

Acceptance 03-06-2021,

Published 02-08-2021.

Copyright (C) 2021 Anvesh Apkari et al. This is an open access article distributed under Creative Commons Attribution License [Attribution 4.0 International (CC BY 4.0)] 


\section{BACKGROUND}

Tuberculosis which was known as a lethal disease prior to the development of antibiotics, before the 1950s had shown a steady fall in incidence thereafter. However, the disease continued to be endemic in few of the developing countries due to malnutrition and drug-resistant strains. In addition, a resurgence of the disease was noticed in developing countries due to conditions like HIV or chronic diseases like DM, chronic renal failure, COPD, cirrhosis of the liver, leukemia, and lymphoma. The growing elderly population, alcohol and drug abuse further contributed to this resurgence. Tuberculosis is both pulmonary and extrapulmonary caused by bacillus Mycobacterium tuberculosis. This has infected about a quarter of the world's population. According to the WHO, for the year 2018 India with a share of $27 \%$ accounted for the country with the highest global TB burden. ${ }^{1}$

Tuberculosis of the central nervous system [CNS] is the most severe form and is the most important cause of morbidity and mortality in affected individuals. It comprises $10 \%$ of total tuberculosis cases and $20 \%$ of TB cases in immuno compromised patients. ${ }^{3,4}$ In most of the cases, the causative organism is $M$. tuberculosis. The hematological spread is the most common mode of spread of the infection to the CNS usually from a pulmonary focus. Direct spread from the paranasal sinuses, mastoids, or orbit has also been reported in a few cases. ${ }^{5}$ Upon reaching the CNS the organism initiates granulomatous inflammatory response which is usually appreciated on magnetic resonance imaging (MRI), which helps in early diagnosis and treatment.

Central nervous system tuberculosis affects the neural tissues and the meninges. This occurs in all age groups, but 60 - $70 \%$ of patients are below the age of 20 years. Intracranial tuberculosis can be meningeal, parenchymal pattern, and also a combination of these patterns. Meningeal tuberculosis includes leptomeningitis and pachymeningitis whereas Parenchymal tuberculosis includes tuberculoma, abscess, cerebritis, rhomboencephalitis, and encephalopathy. ${ }^{3}$

Contrast enhanced MRI is the imaging procedure of choice, as CT scans may be normal in the early stages. However, its limitations include availability as well as the need for sedation in children. TB meningitis is an important clinical aspect of intracranial TB. MRI has high sensitivity with satisfactory specificity in identifying meningeal and parenchymal infections. Signal changes are seen early in the development of the disease, when no other image modality show lesions especially fluid attenuated inversion recovery (FLAIR) and diffusion-weighted imaging [DWI] sequence detects initial infective focus as inflammatory edema. MRI plays an important role in its early diagnosis as well as to demonstrate its complications such as infarcts, the incidence of which to be detected by CT varies from 20.5 to $38 \%$ and is much lower as compared to its ability to be detected by MRI. ${ }^{4}$

However, MRI is not specific for infection. Many noninfectious inflammatory diseases, degenerative diseases, and pyogenic infections mimic intracranial TB. Hence the diagnosis remains challenging. Therefore, there must be a sense of familiarity with its various patterns, which will enable us to make an early and accurate diagnosis, thereby reducing the disease burden. The varied presentation, serious complications, and association with immunodeficiency and chronic health diseases warrant its inclusion in the differential diagnosis in intracranial disorders.

With this background, our study was conducted to evaluate the role of MRI in diagnosing intracranial tuberculosis and study various patterns of its presentations.

\section{METHODS}

This is a single-center, hospital based, observational and retrospective study. MRI findings of 25 patients with diagnosed intracranial tuberculosis were reviewed from March 2015 to April 2020. These patients had been referred to the Department of Radiodiagnosis with clinical suspicion of intracranial pathology. Infection was proven in all patients with positive culture or histological examination.

\section{Ethical Statement}

The study was conducted after obtaining approval from the institutional review board to review the patients' images and medical charts. Basic demographic data of the patients were noted.

\section{MRI Technique}

All patients underwent MRI evaluation on a 1.5 Tesla MR machine (Siemens Magnetom Avanto) using the following sequences T1W, T2W, FLAIR, DWI, and SWI sequences in various planes and post-contrast sequences in orthogonal planes after injecting intravenously with $0.2 \mathrm{ml} / \mathrm{kg}$ body weight of gadolinium based paramagnetic contrast (Magnevist $-0.5 \mathrm{mmol} / \mathrm{ml}$ ). ${ }^{1} \mathrm{H}$ proton spectroscopy was done in atypical cases as well as in cases that provided a diagnostic challenge.

\section{Imaging Evaluation}

Two radiologists independently reviewed the MR images in consensus. The following parameters were studied. The presence of tuberculomas / granulomas, abnormal leptomeningeal and patchy meningeal enhancement, meningioencephalopathy, abscess, hydrocephalus, and infarcts were included.

The data was analyzed and the results were presented using the tables.

\section{RESULTS}

\section{Demographic Data}

The study included 15 males (60\%) and 10 females (40\%), with a male to female ratio of $3: 2$. Most common age group was 21 to 40 years with 10 patients ( $40 \%$ ), followed by 41 to 60 years with 9 patients (36\%). 3 patients each (12\%) were in the age group below 20 years and above 61 years. Most common presenting symptom observed was head ache in 14 patients ( $56 \%$ ) followed by neck pain in 6 patients $(24 \%)$ and low back ache in 3 patients (12\%). Other symptoms observed were fever, functional disability, malaise and weight loss. 

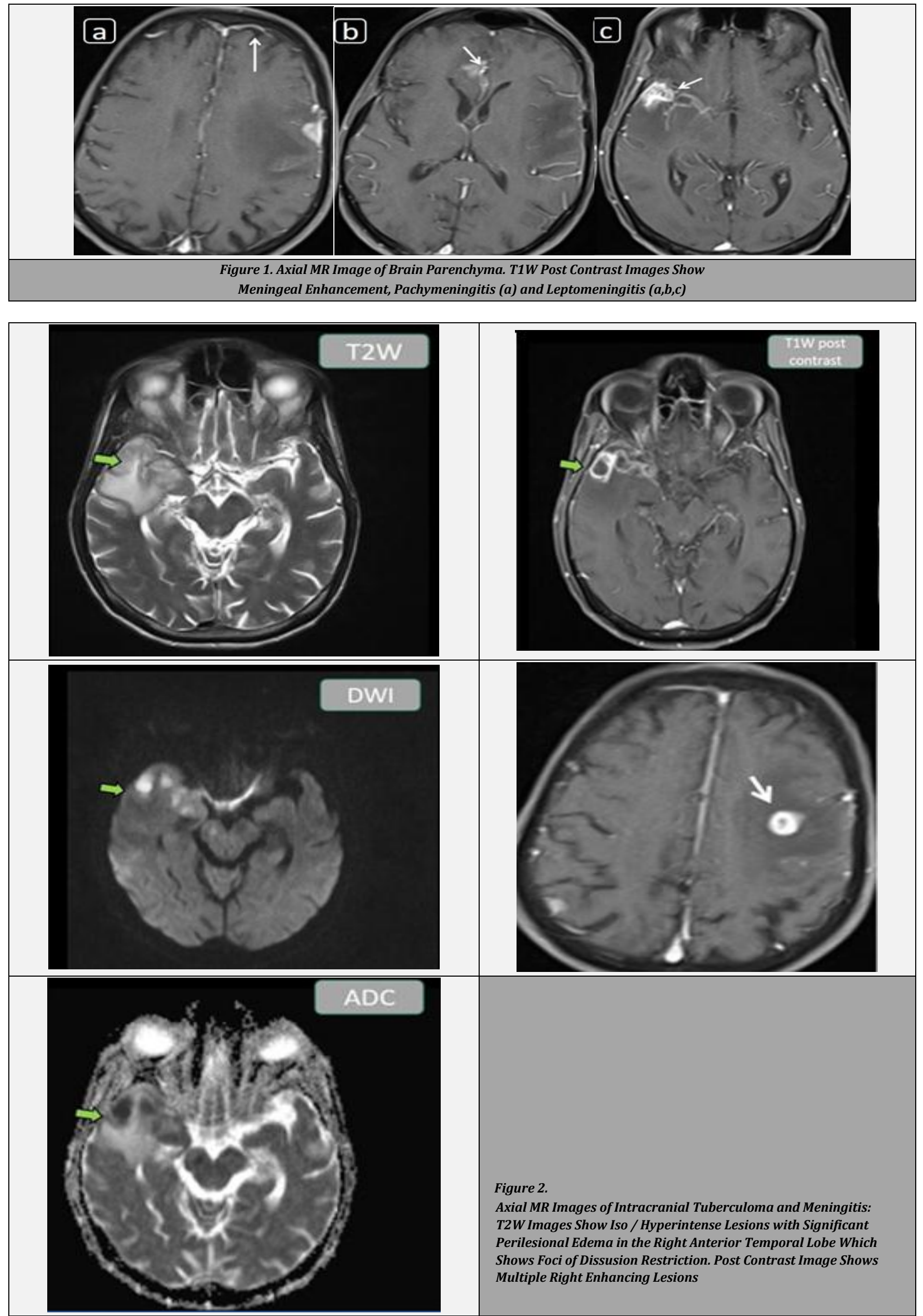

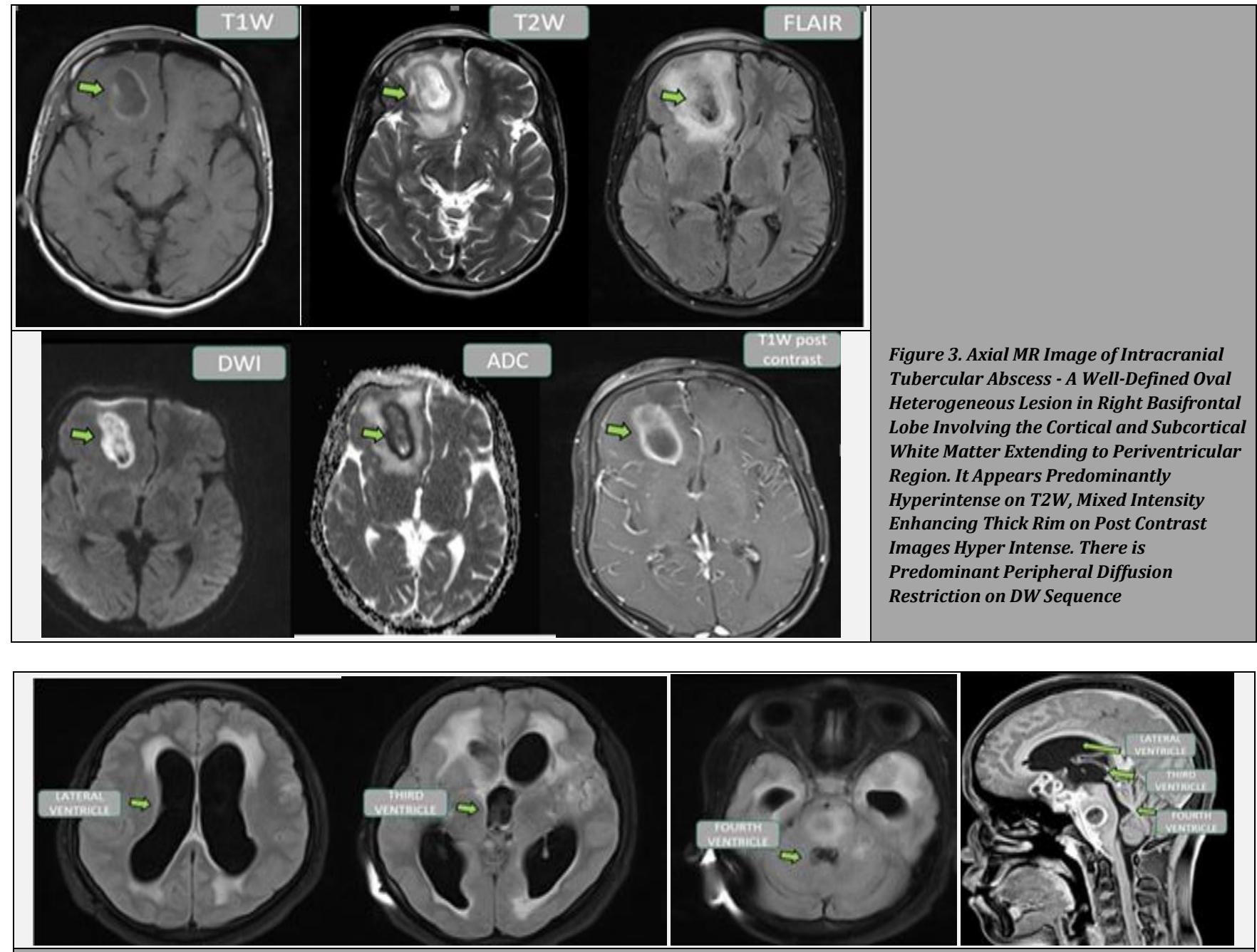

Figure 4. Intracranial Tuberculosis with Complications - Axial and Sagittal MR Images of Brain Showing Hydrocephalus and TB Meningitis. Multiple Rim Enhancing Meningeal Lesions (Recemose Pattern of Meningitis) and in the Pons. Bilateral Dilated Lateral Ventricles Third and Fourth Ventricle

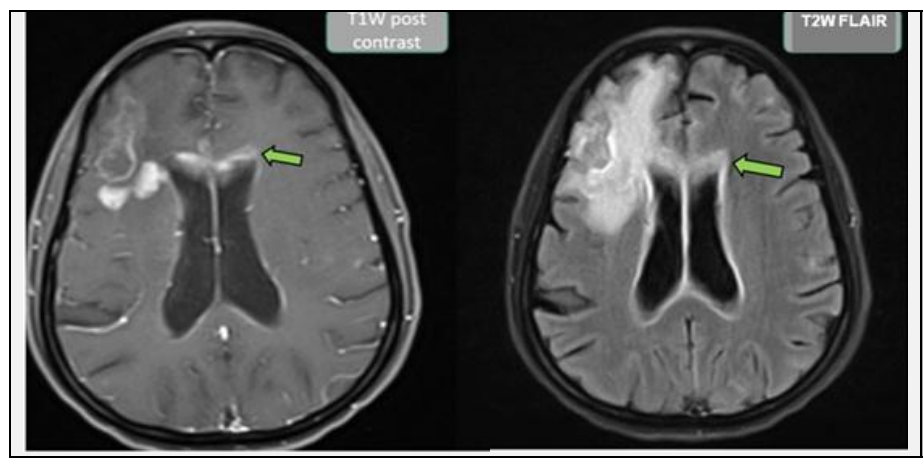

Figure 5. Intracranial Tuberculosis with Complications. MR Images Show Intracranial Tubercular Abscess, Tuberculoma and Ventriculitis. Post Contrast and Flair Axial Images Show Thickened and Hyper-Enhancing Ventricular Lining in Patient with Right Frontal Abscess and Adjacent Nodular Enhancing Lesions

\section{MRI Findings}

Isolated meningeal involvement - Pachymeningitis 2 (8\%). Isolated parenchymal involvement - Tuberculomas 15 (60\%), Abscess 1 (4\%). Combinations of meningitis and tuberculomas in $4(16 \%)$ and meningoencephalitis and tuberculomas in 3 (12\%). Lesions of tuberculoma were seen in 22 patients. Out of these 22 cases, infratentorial involvement was seen in 4 cases. Isolated tuberculoma was the most common pattern of presentation observed. Meningeal enhancement was seen in 9 cases. These included pachymeningitis and leptomeningitis in 2 patients [Figure1] and meningoencephalitis [Figure 2] in 3 cases. Abscess [Figure 3] was seen in one case. Complications of intracranial tuberculosis were observed in 5 patients. Among this hydrocephalus was seen in 4 cases [Figure 4] and infarcts in 2 cases. The infarcts were seen in the gangliocapsular region with one of them showing hemorrhagic transformation and another acute infarct in the right half of the midbrain. Ventriculitis was seen in one case [Figure 5].

\section{DISCUSSION}

Central nervous system tuberculosis is the most devastating clinical manifestation of tuberculosis which is the most severe form of extrapulmonary tuberculosis with high mortality and morbidity. This can be divided into intracranial TB and 
intraspinal TB. ${ }^{5}$ Mycobacterium tuberculosis is responsible for most tuberculous infections of the CNS. During the initial stage of hematogenous dissemination, tuberculomas containing $M$. tuberculosis [rich foci] deposited in the meninges and brain parenchyma rupture and result in the infection. ${ }^{6}$

There are two theories regarding the development of tuberculous lesions. Firstly, in the leptomeninges, rich foci develop due to hematologic spread which ruptures into the subarachnoid space. A second theory is that there is an inflammatory breakdown of the capillary and venule walls as well as the vessels penetrating the cerebrum and myelum due to hematogenous spread. This allows the spread of the infection both superficially over the leptomeninges as well as deeper into the brain and the spinal cord substance. ${ }^{7}$

Intracranial TB presents in various patterns. They are meningeal TB and parenchymal TB. Tubercular meningitis is the most common type of presentation. The other types include tubercular encephalopathy, tuberculomas, and tubercular abscess. ${ }^{6}$ TB has no sex predilection but some studies have shown a male predominance. ${ }^{8}$ as noted in ours.

\section{Meningeal TB}

Tubercular meningitis is the most common presentation of CNS tuberculosis seen in children and adolescents.8,9 This is commonly due to the hematogenous spread of Mycobacterium tuberculosis. Other types of spread include direct extension and / or rupture of a subpial or subependymal 'rich focus', either to the subarachnoid spaces or into the ventricular system. ${ }^{10}$

TB meningitis usually has an insidious course with a nonspecific clinical presentation in the early stages, especially in children. The most sensitive feature of tubercular meningitis is the meningeal enhancement which is found in 90 $\%$ of the cases. ${ }^{11}$ Post-contrast and FLAIR imaging better demonstrates leptomeningeal enhancement. ${ }^{12}$ On MRI, enhancing exudates in the basal cisterns are the most common and specific manifestations of leptomeningeal TB. ${ }^{13}$

Pachymeningitis occurs due to chronic tuberculous infection of the dura. This is seen as a focal or diffuse enhancement of the dura and arachnoid. Meningoencephalitis occurs when the tuberculous exudates extend into the brain parenchyma. Our study results showed a fewer number of meningeal patterns of presentation [24\%], wherein isolated meningitis cases were $8 \%$ and with parenchymal disease they were $16 \%$. [Figure 1 and 2]

\section{Parenchymal TB}

Tuberculomas can occur isolated or in association with tubercular meningitis. They are the most common forms of parenchymal TB. Other parenchymal patterns include cerebritis, cerebral abscess, miliary tuberculosis, or tuberculosis encephalopathy.

Tuberculomas can be solitary or multiple and can occur anywhere in the brain but are commonly located in the corticomedullary junction and the periventricular region. ${ }^{14}$ Tuberculomas appear hypo or isointense to the brain on T1W and hypointense on $\mathrm{T} 2 \mathrm{~W}$ not showing diffusion restriction. Some of the liquefied areas are T2W hyperintense with a hypointense rim and show diffusion restriction. The enhancement is variable but predominantly shows ring enhancement [Figure 2 and 3]. It is important to distinguish tuberculomas from neurocysticercosis, abscess, and neoplasm. MRS can play a role in this. A large lipid peak is seen in $85-90 \%$ of the patients. A prominent decrease in NAA: $\mathrm{Cr}$ with a modest decrease in NAA: Cho is typical. ${ }^{15}$

In our study tuberculomas were the most common [88 \%] presentation. Among these isolated presentations were $60 \%$ and $28 \%$ along with other patterns. This differs from the study conducted by Synmon et al. ${ }^{6}$ where tuberculomas were seen in $28.6 \%$ of the cases.

A tubercular abscess is commonly seen in immunocompromised as well as in elderly patients. TB abscess appears large, multiloculated with ring enhancement and perilesional edema [Figure 4]. They are similar in appearance to pyogenic abscess but they show an amino acid peak at $0.9 \mathrm{ppm}$ which is absent in Tb abscess. ${ }^{16}$

Our study showed the lowest number of cases of abscess, i.e. $4 \%$ similar to the $2.9 \%$ reached by Synmon et al. ${ }^{6}$

\section{Complications}

Hydrocephalus is the most common complication of tuberculous meningitis. It can be communicating or noncommunicating. Communicating hydrocephalus usually occurs secondary to cerebrospinal fluid flow in the basal cisterns. Non-communicating hydrocephalus can occur due to obstruction by tuberculomas. ${ }^{12}$ In our study out of the four cases with hydrocephalus, two cases were associated with meningitis, one with meningoencephalitis, and one with tuberculomas [Figure 4].

An ischemic infarct is another complication that is seen mostly in the basal ganglia or the internal capsule region. The cause being vascular compression and occlusion of small perforating vessels such as the thalamoperforating and the lenticulostriate arteries. ${ }^{17}$ Two cases of infarcts presented in our study were seen in these regions.

Ventriculitis a rare complication can be lethal.18 In our study, it was seen in a single patient who also had tuberculomas, meningoencephalitis, hydrocephalus, and a subacute infarct in the left basal ganglia [Figure 5]. The presence of intraventricular debris, enhancing ventricular wall, and / or enhancement and swelling of the choroid plexus are the general radiological features of Ventriculitis. ${ }^{19}$ The features which could suggest the possibility of a tubercular nature of the ventriculitis are intraventricular septations, sequestration of the ventricles, and a hyperintense ependymal wall on magnetization transfer images on MRI.20

\section{CONCLUSIONS}

MRI is the established modality of choice to diagnose and monitor intracranial tuberculosis. Due to the higher incidence of tuberculomas in our study compared to the existing literature, emphasis should be put on this as an important presenting feature. Good thorough knowledge of the spectrum of intracranial tuberculosis is essential for an early and accurate diagnosis, thereby reducing the disease burden.

Data sharing statement provided by the authors is available with the full text of this article at jemds.com.

Financial or other competing interests: None. 
Disclosure forms provided by the authors are available with the full text of this article at jemds.com.

\section{REFERENCES}

[1] WHO. Global tuberculosis report. World Health Organization 2019. [cited 2020 Apr 30]. http://www.who.int/tb/publications/global_report/en/

[2] Bernaerts A, Vanhoenacker FM, Parizel PM, et al. Tuberculosis of the central nervous system: overview of neuroradiological findings. Eur Radiol 2003;13(8):187690.

[3] Khatri GD, Krishnan V, Antil N, et al. Magnetic resonance imaging spectrum of intracranial tubercular lesions: one disease, many faces. Pol J Radiol 2018;83:e524-35.

[4] Trivedi R, Saksena S, Gupta RK. Magnetic resonance imaging in central nervous system tuberculosis. Indian J Radiol Imaging 2009;19(4):256-65.

[5] Marais S, Thwaites G, Schoeman JF, et al. Tuberculous meningitis: a uniform case definition for use in clinical research. Lancet Infect Dis 2010;10(11):803-12.

[6] Synmon B, Das M, Kayal AK, et al. Clinical and radiological spectrum of intracranial tuberculosis: a hospital based study in Northeast India. Indian J Tuberc 2017;64(2):10918.

[7] McGuiness F. Imaging of brain and spinal cord tuberculosis. In: Madkour MM, ed. Tuberculosis. Berlin, Heidelberg: Springer 2004:547-86.

[8] Fazel PA, Makki KU, Haroon H, et al. Clinical spectrum and outcome of patients with tuberculous meningitis. Med Channel 2006;12(4):21-3.

[9] Raviglione MC, Snider DE Jr, Kochi A. Global epidemiology of tuberculosis: morbidity and mortality of a worldwide epidemic. JAMA 1995;273(3):220-6.
[10] Andronikou S, Wilmshurst J, Hatherill $\mathrm{M}$, et al. Distribution of brain infarction in children with tuberculous meningitis and correlation with outcome score at 6 months. Pediatr Radiol 2006;36(12):1289-94.

[11] Dastur DK, Manghani DK, Udani PM. Pathology and pathogenetic mechanisms in neurotuberculosis. Radiol Clin North Am 1995;33(4):733-52.

[12] Taheri MS, Karimi MA, Haghighatkhah H, et al. Central nervous system tuberculosis: an imaging-focused review of a reemerging disease. Radiol Res Pract 2015;2015:202806.

[13] Parmar H, Sitoh YY, Anand P, et al. Contrast-enhanced flair imaging in the evaluation of infectious leptomeningeal diseases. Eur J Radiol 2006;58(1):89-95.

[14] Andronikou S, Smith B, Hatherhill M, et al. Definitive neuroradiological diagnostic features of tuberculous meningitis in children. Pediatr Radiol 2004;34(11):87685.

[15] Gupta R. Magnetization transfer MR imaging in central nervous system infections. Indian J Radiol Imaging 2002;12(1):51.

[16] Osborn AG, Hedlund GL, Salzman KL. Osborn's Brain. $2^{\text {nd }}$ edn. Elsevier Health Sciences 2017: p. 1388.

[17] Shah GV. Central nervous system tuberculosis: imaging manifestations. Neuroimaging Clin N Am 2000;10(2):355-74.

[18] Kumar S, Kumar R, Radotra BD, et al. Tubercular ventriculitis: an uncommon entity. Indian J Pediatr 2014;81(6):608-10.

[19] Fujikawa A, Tsuchiya K, Honya K, et al. Comparison of MRI sequences to detect ventriculitis. Am J Roentgenol 2006;187(4):1048-53.

[20] Singh P, Paliwal VK, Neyaz Z, et al. Clinical and magnetic resonance imaging characteristics of tubercular ventriculitis: an under-recognized complication of tubercular meningitis. J Neurol Sci 2014;342(1-2):13740. 